\title{
Anomalous optical and electrical recovery processes of the photoquenched EL2 defect produced by oxygen and boron ion implantation in gallium arsenide
}

\author{
J. Samitier, S. Marco, A. Pérez-Rodriguez, and J. R. Morante \\ L. C. M. M. Departament de Fisica Aplicada i Electrònica, Universitat de Barcelona, \\ Avda. Diagonal 645-647, Barcelona 08028, Spain \\ P. Boher and M. Renaud \\ Laboratoire d'Elecironique Philips, 3 Avenue Descartes, BP 15-94450, Limeil-Brevannes, France
}

(Received 30 July 1991; accepted for publication 24 September 1991)

The optical and electrical recovery processes of the metastable state of the EL2 defect artificially created in $n$-type $\mathrm{GaAs}$ by boron or oxygen implantation are analyzed at $80 \mathrm{~K}$ using optical isothermal transient spectroscopy. In both cases, we have found an inhibition of the electrical recovery and the existence of an optical recovery in the range 1.1-1.4 eV, competing with the photoquenching effect. The similar results obtained with both elements and the different behavior observed in comparison with the native EL2 defect has been related to the network damage produced by the implantation process. From the different behavior with the technological process, it can be deduced that the electrical and optical anomalies have a different origin. The electrical inhibition is due to the existence of an interaction between the EL2 defect and other implantation-created defects. However, the optical recovery seems to be related to a change in the microscopic metastable state configuration involving the presence of vacancies.

\section{INTRODUCTION}

It is well known that the EL2 defect in GaAs presents an interesting optical property known as the photoquenching effect. Basically, this feature is attributed to the existence of two different atomic configurations, labeled stable and metastable, for the same charge state of the defect. The main characteristic of the metastable state is that it does not show optical response when the sample is irradiated with photons in the energy range about $0.9-1.4 \mathrm{eV}$ at temperatures below $120 \mathrm{~K}$ (for a review of the EL2 properties see Refs. 1 and 2). This behavior has been observed by different experimental techniques, such as photoluminescence, photoconductivity, photocapacitance, or infrared absorption and has been suggested as an accurate method to determine the presence of the EL2 defect in the gallium arsenide as-grown or after irradiation or implantation processes. $^{3}$

Two mechanisms have been proposed by different authors ${ }^{1,2}$ in order to achieve the complete transfer from the metastable to stable configuration: (i) thermal mechanism, with an activation energy of about $0.3 \mathrm{eV}$; (ii) Electrical mechanism, which is induced by free carriers injection. Moreover, a third partial recovery mechanism has been reported by several authors when the samples are optically irradiated with photons of given energies. ${ }^{47}$ However, these works disagree in some important aspects such as the amount of optical recovery and their spectral dependence.

These differences, in addition to other discrepancies observed in the characteristic parameters of the EL2 center, measured by several techniques from different samples, have led to the concept of the EL2 family as a family of similar defects with different microscopic configurations. ${ }^{4,8}$
We have to remark that, in spite of many works devoted to the EL2 defect, its microscopic structure is unknown, although it is generally assumed that the EL2 involves the arsenic antisite defect, $\Lambda s_{\mathrm{Ga}}$, as the main ${ }^{9,10}$ or exclusive component. ${ }^{11-13}$ The existence of defect interactions between an essential EL2 and other structural defects or impurities has been suggested to explain the discrepancies observed in the defect properties. In this way, Manasreh and Fisher ${ }^{8}$ have recently pointed out that differences in the photoinduced recovery process of the metastable state can be related to the presence and nature of other defects and impurities surrounding the EL2 defect. Likewise, Parker and Bray ${ }^{14}$ have claimed that the variety of thermal recovery processes in different samples can be explained by the possibility that metastable defects can exist with structural modifications, possibly related to the impurities, and Desnika, Desnika, and Santic, ${ }^{15}$ from the analysis of the photoconductivity and thermally stimulated current in semi-insulating (SI) GaAs, suggest EL2 as a gettering center for other native defects or impurities.

On the other hand, although the EL2 is a native defect in the material grown by Bridgman and Czochralski techniques, different authors have reported the artificial creation of deep defects with characteristics very similar to those of the EL2 center by irradiation of neutrons or implantation of different ions $(\Lambda \mathrm{s}, \mathrm{Ga}, \mathrm{O}, \mathrm{Ne}, \mathrm{B})$ followed by high-temperature thermal annealing $\left(T>600^{\circ} \mathrm{C}\right) .{ }^{16-18} \mathrm{In}$ these cases, the activation energies of the emission rate and the photoquenching spectra are also found to show differences in relation to the starting EL2 defect. These depend on the characteristics of the starting material, the implanted specimen, and the annealing treatment. More recent works have reported the strong ability of the EL2 
center created by implantation processes for its interaction with shallower defects, questioning the need for a thermal treatment for the creation of the defect. ${ }^{19}$

Therefore, an interesting approach to achieve a clearer understanding of the nature of the EL2 defect can be obtained from the analysis of the properties of the EL2-like centers produced by ion implantation, as well as their interaction with shallower defects also created during these processes. In this framework, we present the results on the characterization of the photoquenching recovery of the EL2 center created by means of the ion implantation of two different elements, oxygen and boron. The analysis has been done in a comparative way in relation to the behavior observed in as-grown material, where EL2 is a native defect.

\section{SAMPLES AND EXPERIMENTAL DETAILS}

Oxygen-implanted samples have been obtained using as starting material liquid-encapsulated Czochralsky (LEC) GaAs Se doped with a mean free carrier concentration of $10^{17} \mathrm{~cm}^{-3}$ and an EL2 native concentration of about $N_{T}=10^{14} \mathrm{~cm}^{-3}$. The samples were implanted with an energy of $100 \mathrm{keV}$ and doses of $10^{10}$ and $10^{11} \mathrm{~cm}^{-2}$. For the boron-implanted samples, in order to avoid possible effects of residual boron contamination observed sometimes in LEC ingots, we have used as starting material horizontal Bridgman $n$-GaAs Te doped with a free electron concentration of about $8 \times 10^{16} \mathrm{~cm}^{-3}$. In this case, before the implantation, the wafers were annealed at $870^{\circ} \mathrm{C}$ for 15 min with a $\mathrm{Si}_{3} \mathrm{~N}_{4}$ capping layer to decrease the EL2 native concentration to $N_{T}=10^{14} \mathrm{~cm}^{-3}$ in a $1-\mu \mathrm{m}$-thick layer below the surface. The samples were implanted with boron with an energy of $100 \mathrm{keV}$ and dose $10^{11} \mathrm{~cm}^{-2}$.

After implantation, some samples were annealcd at temperatures between $300^{\circ} \mathrm{C}$ and $500^{\circ} \mathrm{C}$ in an open furnace under $\mathrm{H}_{2}$ flux for $15 \mathrm{~min}$ using the close-contact technique to prevent arsenic out-diffusion. Schottky junctions were obtained by gold evaporation on the surface, with a contact area of $0.64 \mathrm{~mm}^{2}$. The ohmic contacts were made in the back of the samples with tin.

The optical characterization of the metastable state of the EL2 and its recovery mechanism have been carried out by means of the analysis of the voltage transients obtained under constant capacitance conditions. To analyze the optical induced transients, we have used the optical isothermal transient spectroscopy (OITS). The signal transient $S(t)$ can be described in terms of a time constants distribution function $G(\tau)$ as:

$$
S(t)=A\left[1-\int_{0}^{\infty} G(\tau) \exp \left(-\frac{t}{\tau}\right) d \tau\right]
$$

where $A$ is proportional to the deep trap concentration $N_{T}$. The OITS signal for each time $t_{i}$, OITS $\left(t_{i}\right)$, is given by sampling the photocapacitance transient at different times $t_{i} / \sqrt{K}$ and $t_{i} \sqrt{K}$, where $K$ is a fixed parameter, by means of the following expression:

$$
\operatorname{OITS}\left(t_{i}\right)=\frac{S\left(t_{i} / \sqrt{K}\right)-S\left(t_{i} \sqrt{K}\right)}{\ln K} .
$$

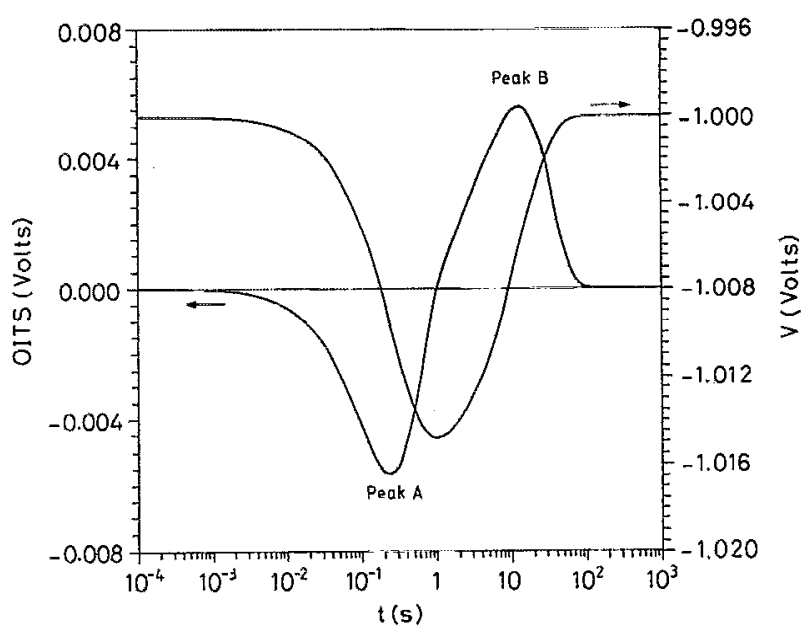

FIG. 1. Voltage transient and the OITS spectrum for as-grown GaAs (LEC) under $1.1 \mathrm{eV}$ illumination at $T=80 \mathrm{~K}$.

It can be shown that the OITS signal of an arbitrary transient corresponds to a convolution product between the time constant distribution of the transient and the OITS signal of a purely exponential one. ${ }^{20}$ In this case the OITS spectrum has a characteristic shape and it exhibits a maximum located at:

$$
t=\tau \frac{\sqrt{K} \ln (K)}{K-1}
$$

with an amplitude given by

$$
\text { OITS }_{\max }=A \frac{K-1}{e \ln (K)} K^{-[K /(K-1)] .}
$$

In this way, the OITS technique allows us to determine for an optical transient its time constant distribution function. It is advantagcous over the deep level optical spectroscopy (DLOS) ${ }^{21,22}$ because for this last technique the signal obtained for each photon energy corresponds to the contribution of all the levels with optical response. Then, to separate each contribution it is necessary to carry out DLOS from different initial occupancy factor values. In addition, the application of this technique for complex systemswith nonexponential or multiexponential processes-may lead to misinterpretation of the experimental data. $\mathrm{On}^{-}$the other hand, the experimental implementation of the OITS method is easier than that of the multi-exponential analysis methods based on the Fourier transform ${ }^{23,24}$ or the method of moments. ${ }^{25}$ The experimental setup used has been reported elsewhere. ${ }^{26}$

\section{RESULTS}

Figure 1 shows the $1.1 \mathrm{eV}$ optically induced transient and the corresponding OITS spectrum, obtained at constant capacitance from an LEC as-grown sample. The OITS spectrum has two peaks, one negative (labeled $\mathrm{A}$ ) and the other positive (labeled B), of equal area corresponding to two exponential components of the transient. The B peak corresponds to the optical quenching associ- 


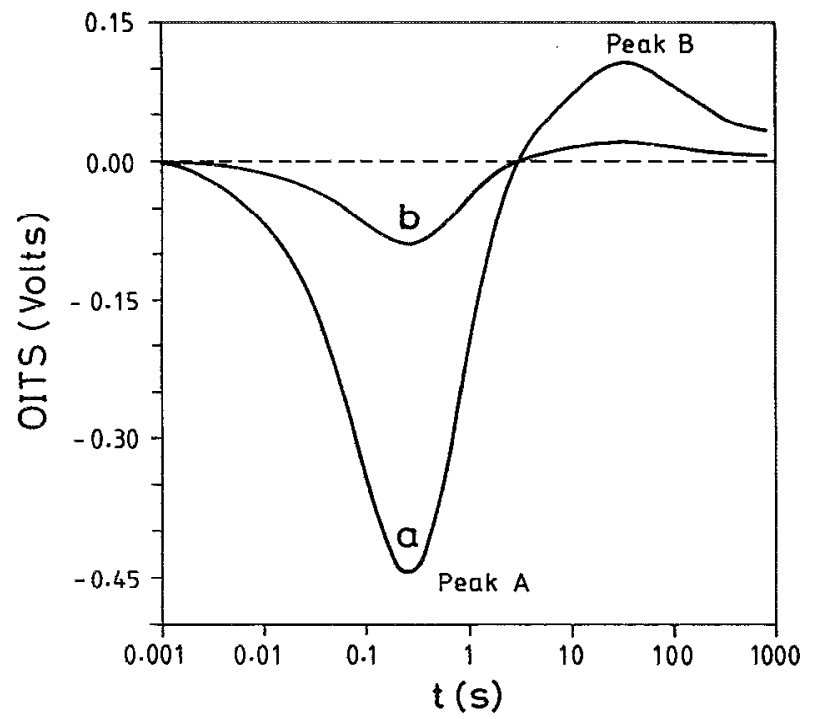

FIG. 2. OITS spectra under $1.1 \mathrm{eV}$ illumination at $T=80 \mathrm{~K}$ for oxygen as-implanted samples: (a) dose $10^{11} \mathrm{~cm}^{-2}$, (b) dose $10^{10} \mathrm{~cm}^{-2}$.

ated with the EL2 level. Figure 2 shows the OITS spectra with an illumination energy of $1.1 \mathrm{eV}$ from as-implanted $10^{10}$ and $10^{11} \mathrm{~cm}^{-2}$ oxygen samples. The great increase of the quenching amplitude (B peak) from as-implanted oxygen samples compared to that from the as-grown one points out the creation of EL2-related centers without a high-temperature annealing of the samples and corroborates previous results reported by thermal and optical techniques in boron-implanted samples. ${ }^{19,27,28}$

Unlike the as-grown samples, where the $A$ and $B$ peaks present the same areas, ${ }^{21}$ in the oxygen-implanted samples we observe a strong difference between both areas, being always the area of $\mathrm{A}$ peak greater than that of the $\mathrm{B}$ one. For the boron-implanted samples the spectra show a similar behavior, as can be observed in Fig. 3(a), where we present the OITS spectra obtained from the $10^{11} \mathrm{~cm}^{-2}$ boron-implanted sample and annealed at $400^{\circ} \mathrm{C}$.

In the case of boron implantation, these differences have been partially explained taking into account the previously proposed interaction model $1^{27,28}$ between the EL2 defect and shallower levels created during the implantation processes, identified as the EL6 and EL5. ${ }^{19,29}$ According to this model, the photoionization of these levels takes place through the EL2 one, previously ionized, which leads to an increase of the A peak amplitude in relation to that of the $B$ one. This effect has been observed performing optical transients which these shallower levels previously empticd by thermal emission ( $180 \mathrm{~K}$ for $20 \mathrm{~min}$ ). The results (Figs. 3 and 4 ) show that only the area of the A peak decreases while the B peak remains unchanged. Nevertheless, only for photon energies equal or below $1.1 \mathrm{eV}$ both $\mathrm{A}$ and $\mathrm{B}$ peaks have the same area.

The oxygen-implanted samples show a similar behavior. Previous results obtained by thermal transients in these samples also present defects with activation energies similar to those of these shallower levels. ${ }^{29}$ The optical tran-

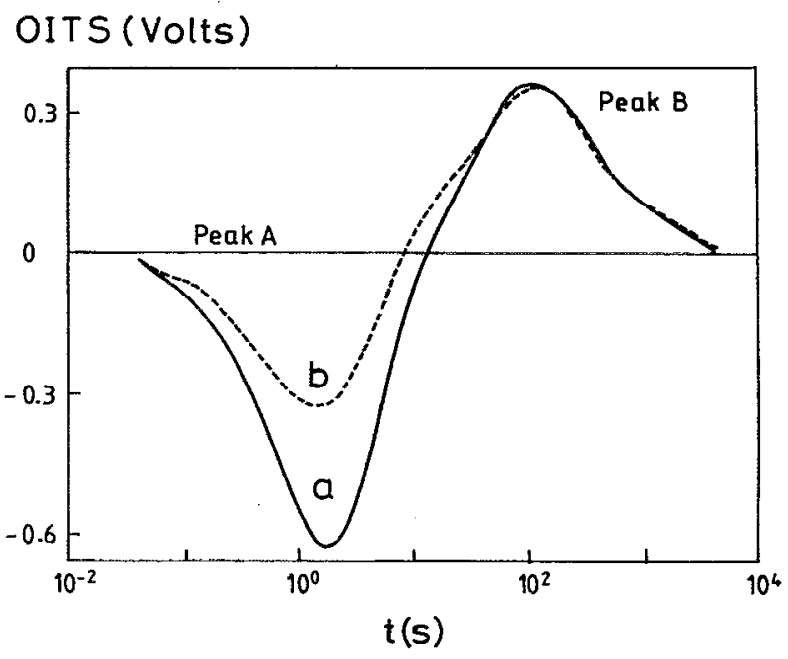

FIG. 3. OITS spectra under $1.1 \mathrm{eV}$ illumination at $T=80 \mathrm{~K}$ for the boron-implanted, $400^{\circ} \mathrm{C}$ annealed sample: (a) first spectrum, (b) spectrum after thermally emptying shallower levels (EL5, EL6) at $180 \mathrm{~K}$ for 20 min.

sients performed after emptying shallower levels, in the same experimental conditions than for the boron-implanted samples (Fig. 5) show a decrease of the area of the A peak in relation to that of the B one. However, in this case the area of the A peak always remains greater than the $B$ one for all illumination energies while the photoquenching effect is present.

To explain the remaining discrepancy between the $\mathrm{A}$ and $B$ peak areas observed in both the oxygen- and boronimplanted samples after emptying the shallower levels two hypothesis can be assumed: (i) there are other deep levels with the same optical ionization rate of the EL2, perhaps EL2 defect without quenching (as has been proposed for the anion antisite defects produced in plastically deformed

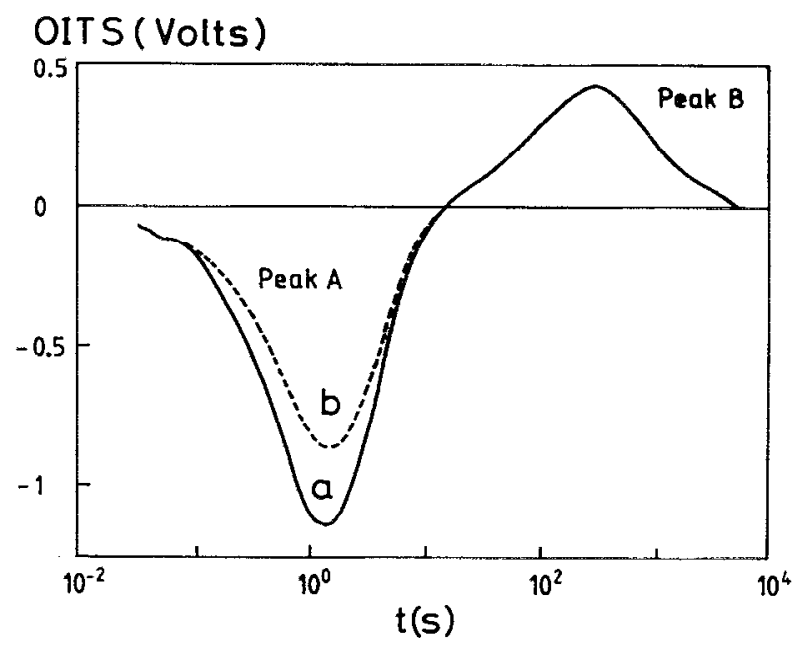

FIG. 4. OITS spectra under $1.2 \mathrm{eV}$ illumination at $T=80 \mathrm{~K}$ for the boron-implanted, $400^{\circ} \mathrm{C}$ annealed sample: (a) first spectrum, (b) spectrum after thermally emptying shallower levels (EL5, EL6) at $180 \mathrm{~K}$ for 20 min. 


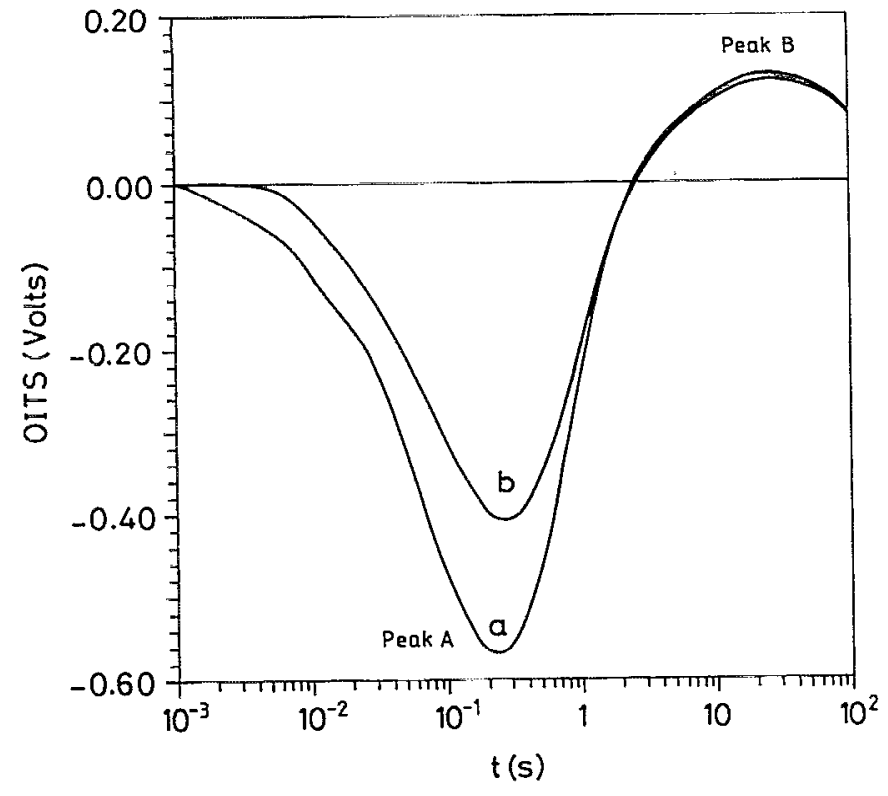

FIG. 5. OITS spectra under $1.1 \mathrm{eV}$ illumination at $T=85 \mathrm{~K}$ for the oxygen $10^{11} \mathrm{~cm}^{-2}$ implanted, $450^{\circ} \mathrm{C}$ annealed: (a) first spectrum, (b) spectrum after thermally emptying shallower levels (EL5, EL6) at $180 \mathrm{~K}$ for $20 \mathrm{~min}$.

$\mathrm{GaAs}^{30}$ or (ii) there is an optical recovery of the metastable state that competes with the transfer from the stable state of EL2. ${ }^{4-6,14}$ In this case the area of the B peak observed will diminish. A third hypothesis, the presence of others levels nonemptied after the thermal emission during $20 \mathrm{~min}$ at $180 \mathrm{~K}$ which could also interact with the EL2, should be taken into account. However, the thermal ionization spectra (DLTS or ITS) from both oxygen- and boron-implanted samples only show a broad peak, the $U$ band, in addition to the EL 6 and EL 5 ones. This band has been associated to an interaction between EL2 and shallower defects. ${ }^{31,32}$ Notice that the existence of a defect with thermal response in the U-band region may be masked by the signal from the $U$ band, making its detection very difficult. Anyway, its feasible existence does not modify the following analysis.

To distinguish between the above hypothesis, a careful analysis of the experimental data is required. Since the optically generated carricrs and/or the Debye tail carriers

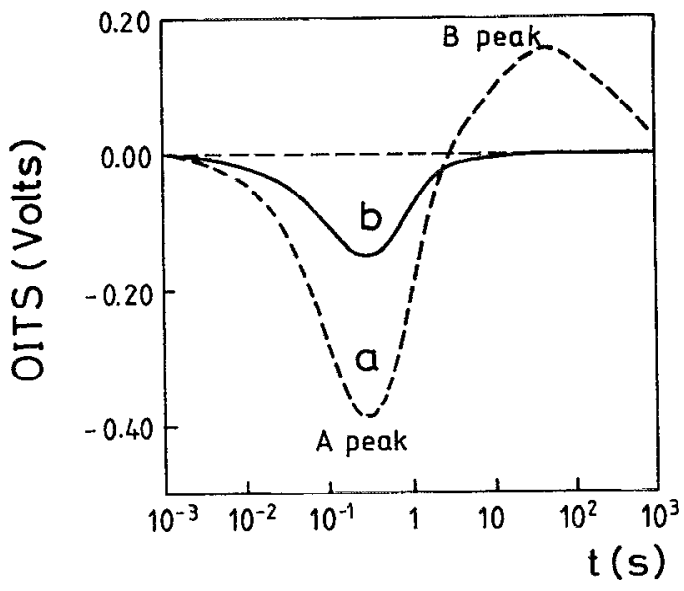

FIG. 6. OITS spectra under $1.1 \mathrm{eV}$ illumination at $T=85 \mathrm{~K}$ for the oxygen as-implanted sample (dose $10^{11} \mathrm{~cm}^{-2}$ ). (a) Dashed line: first spectrum after cooling down, (b) solid line: next spectrum after a forward bias pulse of $1000 \mathrm{~s}$.

can produce an electrical regeneration process, which would be interpretated as an optical recovery, ${ }^{33}$ it is necessary to determine the electrical recovery process present in our samples.

From the measurements made at $80 \mathrm{~K}$, we have observed that the ion-implanted samples do not show a completely electrical recovery of the metastable state. Then, to assure the complete recovery of the EL2 metastable state after each illumination transient, the temperature was increased over $150 \mathrm{~K}$. Table I presents the efficiency of the electrical recovery for implanted samples, obtained from the ratio between the quenching amplitude in the first optical transient after cooling down and the amplitude after a forward bias pulse $1000 \mathrm{~s}$. This time should be enough to obtain the total recovery of the standard EL2 metastable state. This effect appears for both implanted specimens and increases with the implanted dose. Moreover, for the same implantation doses the electrical regeneration rate is greater for the boron-implanted samples. It should be noted that the $10^{11} \mathrm{~cm}^{-2}$ oxygen-implanted sample does not show electrical recovery (Fig. 6).

The failure of the electrical regeneration in uxygenimplanted samples has already been reported by Taniguchi and Ikoma, ${ }^{34}$ but in our case, we note that this effect is not

TABLE I. Efficiency of the electrical recovery for oxygen- and boron-implanted samples. The shallow defects concentration have been measured by thermal transients. For the $10^{11} \mathrm{~cm}^{-2}$ oxygen as-implanted the shallow defect concentration has not been estimated, because the shallow levels peaks were overlapped by the $U$ band.

\begin{tabular}{lcccc}
\hline \hline Element & Dose $\left(\mathrm{cm}^{-2}\right)$ & $\begin{array}{c}\text { Annealing } \\
\text { temperature }\left({ }^{\circ} \mathrm{C}\right)\end{array}$ & $\begin{array}{c}\text { Electrical } \\
\text { recovery }(\%)\end{array}$ & $\begin{array}{c}\text { Shallow defect } \\
\text { concentration }\left(\mathrm{cm}^{-3}\right)\end{array}$ \\
\hline Boron & $10^{11}$ & $\ldots$ & 14 & $5.8 \times 10^{16}$ \\
Boron & $10^{11}$ & 400 & 16.5 & $2.3 \times 10^{16}$ \\
Boron (Ref. 28) & $10^{11}$ & 600 & 87 & $<10^{15}$ \\
Oxygen & $10^{10}$ & $\ldots$ & 13 & $1.0 \times 10^{16}$ \\
Oxygen & $10^{10}$ & 450 & 22 & $3.9 \times 10^{15}$ \\
Oxygen & $10^{11}$ & $\ldots$ & $<1$ & $\ldots$ \\
Oxygen & $10^{11}$ & 450 & $<1$ & $1.0 \times 10^{17}$ \\
\hline
\end{tabular}




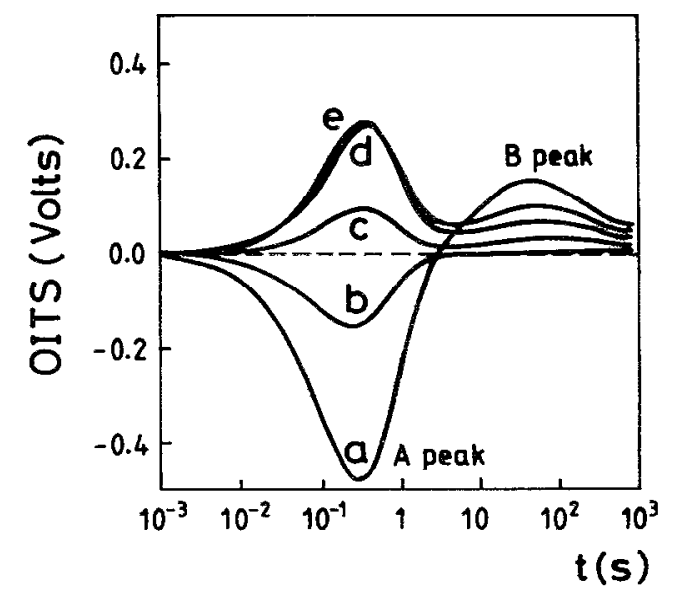

FIG. 7. OITS spectra for the oxygen as-implanted $\left(10^{11} \mathrm{~cm}^{-2}\right)$ at $T=85$ $\mathrm{K}$ under $1.1 \mathrm{eV}$ illumination: (a) initial transient after illuminating 20 min with an excitation energy of: (b) 1 , (c) 1.2 , (d) 1.3 , (e) $1.4 \mathrm{eV}$.

uniquely related to the oxygen samples, because it also appear in boron-implanted samples.

Once determined the inhibition factor of the electrical recovery process, a more accurate analysis of the phototransients has been performed in order to explain the discrepancy between the A and B peak amplitudes after emptying the shallower levels. The experimental sequence has been the following: First, the sample was cooled down to $85 \mathrm{~K}$. Then it was illuminated with a reference energy $h v=1.1 \mathrm{eV}$ during time $t_{1}=25 \mathrm{~min}$, long enough to reach the steady state. Afterwards, the illumination energy was changed to an excitation energy in the range 0.7 to $1.45 \mathrm{eV}$ during a time $t_{2}=25 \mathrm{~min}$. Finally the illumination was returned to $h v=1.1 \mathrm{eV}$ and an OITS spectrum was recorded. This process was repeated for different energies in this excitation range and the sequence has also been done for different reference energies. In all cases, as the optical transients were performed at constant capacitance conditions, the charge space region analyzed was fixed during the experimental process. In Fig. 7 we have plotted the spectra obtained from the as-implanted $10^{11}$ oxygen ions $/ \mathrm{cm}^{2}$ sample. These spectra can only be explained assuming that the optical-induced transition between the metastable and the fundamental state takes place. The first peak in the spectra for the different excitation encrgics is due to a readjustment of the defect occupation factor corresponding to the A peak, between $h v=1.1 \mathrm{eV}$ and the excitation energy used. The second one is due to the quenching transition, carried out at reference energy 1.1 $\mathrm{eV}$, of the EL2 recovered centers from the metastable state. Then, this quenching peak amplitude is a direct measure of the EL2 metastable optical recovery induced for the different excitation energies in relation to the quenching efficiency of the reference energy. This peak cannot be due to a population readjustment of other level, because in such a case it ought to appear also in the spectrum b of Fig. 6, where the EL2 was previously quenched using $h v=1.1$ $\mathrm{eV}$. Moreover, it cannot be related to an electrical regeneration process induced by the presence in the conduction

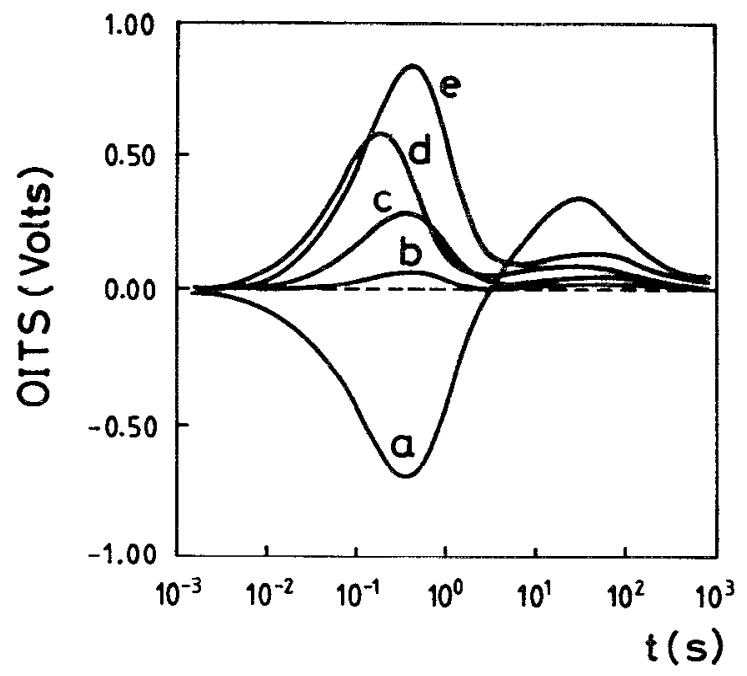

FIG. 8. OITS spectra for the $10^{11} \mathrm{~cm}^{-2}$ boron-implanted and $400^{\circ} \mathrm{C}$ annealed sample at $T=80 \mathrm{~K}$, under $1.1 \mathrm{eV}$ illumination: (a) initial transient after illuminating $20 \mathrm{~min}$ with an excitation energy of: (b) 1.15 , (c) 1.2 , (c) 1.25 , (d) $1.3 \mathrm{eV}$.

band of optically generated carriers, or to an electrical regeneration induced by the free clcctrons in the Debye tail, because we have not observed any electrical regeneration in this sample.

Similar experiments have been performed for the boron- and oxygen-implanted samples which show a partial electrical recovery process (Fig. 8). In Fig. 9 we have plotted the efficiency of the optical recovery versus the illumination energy for the different boron- and oxygenimplanted samples. This efficiency was calculated as the ratio between the quenching amplitude obtained for the reference energy before and after the illumination with ex-

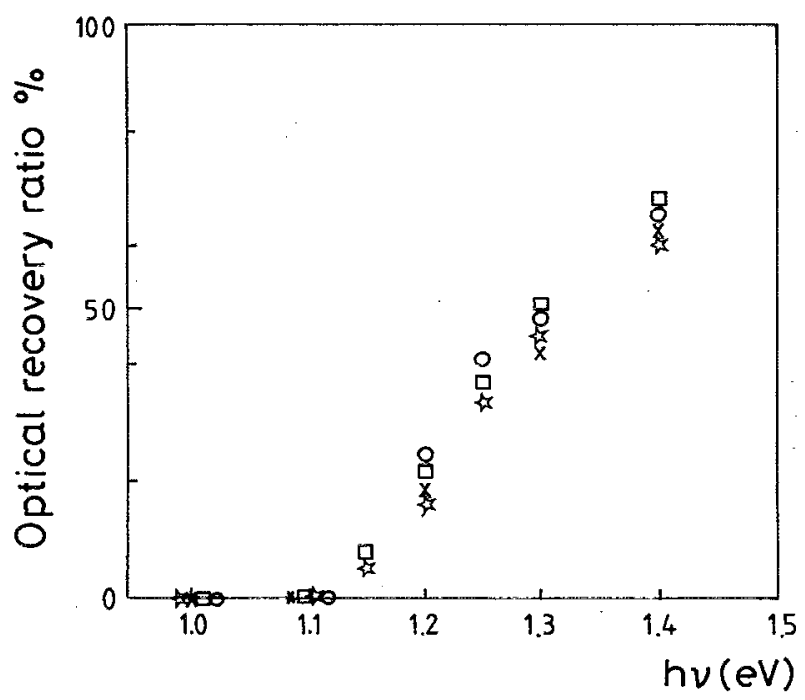

FIG. 9. Spectral dependence of the optical recovery ratio for different samples. $10^{11} \mathrm{~cm}^{-2}$ boron implanted, $400^{\circ} \mathrm{C}$ annealed, $x: 10^{10}$ $\mathrm{cm}^{-2}$ oxygen as-implanted; $\square: 10^{10} \mathrm{~cm}^{-2}$ oxygen implanted, $450^{\circ} \mathrm{C}$ annealed, $O: 10^{11} \mathrm{~cm}^{-2}$ oxygen as-implanted. 
citation energies. We observe a similar evolution for all samples. This result points out that the optical recovery is found to be nearly independent on the boron or oxygen ion specimen nature and the annealing temperature below $500^{\circ} \mathrm{C}$. For reference energies equal to or below $1.1 \mathrm{eV}$ the optical recovery efficiency spectra are identical. It must be noted that for these energies, in boron-implanted samples, the $A$ and $B$ peaks have the same area after emptying the shallower levels. This result suggests that for these energies there is no optical recovery of the metastable state and as a consequence the optical recovery efficiency obtained is independent of the reference energy. Moreover, as the optical recovery evolution is similar for boron and oxygen samples, the differences observed in the areas of the A and $\mathbf{B}$ peaks from the OITS spectra in oxygen-implanted samples after emptying defects could be a possible manifestation of an oxygen-related defect with similar optical response to the EL2 or which can interact with the EL2 center. However, the possibility of an optical recovery at energies equal or below $1.1 \mathrm{eV}$ in the oxygen-implanted samples cannot be discarded.

\section{DISCUSSION}

The obtained results give direct evidence of the presence of optical regeneration of the EL2 centers produced by oxygen and boron implantation. The spectral dependence of this optical recovery (Fig. 9) agrees qualitatively with the previous results reported by Parker and Bray ${ }^{14}$ in oxygen-doped samples, which also observed a photoinduced recovery process between 0.9 and $1.4 \mathrm{eV}$ competitive with the usual photoquenching process. However, they observed a maximum of the spectral efficiency at $1.3 \mathrm{eV}$, and attributed the recovery process to the presence of oxygen modifying the EL2 defect.

Different authors ${ }^{4-6,14}$ have also reported examples of the photoinduced recovery process of the EL2 defect in SI GaAs samples in the optical range between 0.65 and 1.1 $\mathrm{eV}$. Although in this case the recovery process is noncompetitive with the photoquenching effect, only a partial recovery $(10 \%-20 \%)$ is observed. More recently Manasreh and Fisher ${ }^{8}$ have shown a new photoinduced recovery process by photon energies near the GaAs gap ( $h v>1.4 \mathrm{eV}$ ) and with a high recovery ratio. Nevertheless, in all these cases the samples analyzed were as-grown and with a shallow defect concentration very much lower than in our samples.

Unlike these previous results, in our samples the observed electrical and optical recovery processes seem to be related to the implantation damage and the feasible defect interaction. The electrically active shallow defect concentrations shown in Table I are an indication of total defect concentration which is related to the annealing temperature and implantation dose. Both the photoionization amplitude transient of the stable state of EL2 ${ }^{27}$ (peak A in Fig. 2) and the electrical recovery ratio of the metastable state (Table I) strongly depend on the created defects. These seem to indicate that the lattice damage induced by the implantation process modifies the properties of the EL2 defect. In the following, we are going to analyze the relationship between the electrical and optical recovery and the implanted damage.

A first possible explanation of the electrical recovery failure may be based on a diminution of the carriers on the conduction band caused by a compensation process. It is well known that both boron and oxygen implantation can produce a semi-insulating layer in previously $n$-doped GaAs, ${ }^{35}$ which is usually applied to obtain planar device isolation in GaAs integrated circuits.

However, from the measurement of the free carriers profile, we have deduced that the complete electrical recovery of the centers ought to take place in less than $1 \mathrm{~ms}$, and this has not been observed even for forward bias times of the order of hundreds of seconds. Moreover, when the samples are cooled down from room temperature to $80 \mathrm{~K}$ at forward bias and under illumination, the following OITS spectrum, obtained by switching the sample to reverse bias, does not show any photoquenching signal. This fact indicates that it is possible to induce the quenching process even in the semiconductor neutral zone, which is in agreement with the observed inhibition of the electrical recovery process of the quenching state. These data suggest a microscopic origin for the inhibition effects on the electrical recovery process.

Nevertheless, another explanation that assumes that the damage produced by the ion bombardment modifies locally the free carrier concentration is possible. Under these conditions, Makram-Ebeid and Boher ${ }^{36}$ proposed the existence of damage clusters, with a large number of densely packed defects. Across this damaged region, the energy band diagram is modified and the Fermi level is strongly pinned at midgap defects. Then, when we apply a forward bias pulse to reduce the diode depleted region, cluster regions will remain empty of free carriers. If the EL2 defect observed were located in these clusters the capture processes would be slowed by the lower carrier concentration. However, we have observed that in the darkness at $80 \mathrm{~K}$, the EL2 defect presents the normal value of its electron capture cross section.

As a consequence, the failure of the electrical recovery process must be due to the existence of an interaction between the basic EL2 defect and others defects created during the implantation process. The higher inhibition on the electrical recovery process in the oxygen-implanted samples in relation to the boron-implanted ones would be related to the higher damage induced by the heavier implanted ion. This explanation has been corroborated by measurements carried out in annealed implanted samples, which show an increase of the electrical recovery when shallow defects created by implantation vanish. ${ }^{28,29}$

However, for the photoinduced recovery process in the 1-1.4 eV range, we have observed a very weak dependence with the shallower levels concentration (Fig. 9). This process has been observed in the same spectral range by other authors in samples as-grown and oxygen doped, and in other optical ranges in LEC and semi-insulating GaAs. In these samples, the concentration of shallower defects is not significant. Then, we can argue that the photoinduced re- 
covery process has a different origin than the failure of the electrical recovery, more likely related to the microscopic structure of EL2 metastable state.

Taking into account these considerations, as well as the similarities in the photorecovery response in oxygendoped and boron- and oxygen-implanted samples, we think that the changes induced in the basic EL2 defect are similar. It is then necessary to analyze the possible resemblance among the EL2, the oxygen-related defects, and the deep defects produced by implantation.

In fact, the microscopic structure of the oxygen-related centers in GaAs is also not completely known. Absorption measurements have allowed the identification of two bands associated to the isolated oxygen interstitial defect, $\mathrm{O}_{i}$, and a complex defect of arsenic vacancy and oxygen labeled $\mathrm{Ga}-\mathrm{O}-\mathrm{Ga}$ defect. ${ }^{37,38}$ Recently, many works have been devoted to analyze the electrical behavior of these defects. The interstitial oxygen is a nonelectrically active defect, however; Bourgoin et al. ${ }^{39}$ have suggested that the $O_{i}$ interacts with the EL2, changing its DLTS spectrum at temperatures higher than $300 \mathrm{~K}$.

In contrast to the previous results where an oxygenrelated defect labeled ELO shows an activation energy for electron emission similar to the EL2, ${ }^{40}$ Neild, Skowronski, and Lasows ${ }^{41}$ have identified a DLTS peak located at 0.55 $\mathrm{eV}$ below the conduction band as due to the Ga-O-Ga defect. In both cases, the models proposed for the oxygenrelated deep level ${ }^{41,42}$ assume a complex defect formed by an oxygen atom in the site of arsenic and arsenic and/or gallium vacancies.

In a similar way, we should also consider the defects related to the boron atom. Unlike the oxygen atom, which presents an electrically active deep level, previous works have only reported for the boron a shallow double acceptor $B_{\text {As }}{ }^{43}$ Only for implanted boron samples at high doses $>10^{13} \mathrm{~cm}^{-3}$ and annealed at $T>500{ }^{\circ} \mathrm{C},{ }^{44}$ the existence of boron-related complex defects has been suggested.

In consequence, it seems necessary to consider the deep defects due to the ion implantation process, to find the similarity between oxygen and boron effects. In both cases, a significant modification of the lattice order is produced. Many atoms are displaced of their sites and may be placed at interstitial positions. By the assumed microscopic models of EL2, some displaced arsenic atoms will make $\mathrm{As}_{\mathrm{Ga}}$ antisite defects and will be the basis of the created EL2. In the neighborhood of these created defects the crystal network is very perturbed and there exists structural defects such as arsenic and gallium vacancies and interstitial atoms, which can interact, forming complex defects.

Since the optical recovery process is also observed, without noticeable modifications, in the annealed samples $\left(T \leqslant 450^{\circ} \mathrm{C}\right)$, it is not probable that the interstitial atoms would be the origin of this process because they present a high mobility at relatively low temperatures. Therefore, it seems that the vacancies may account for the behavior found. A possible relation between the EL2 and the vacant defects has been previously reported: by theoretical considerations some models propose that the metastable state of the EL2 is the $\mathrm{As}_{i}-\mathrm{V}_{\mathrm{Ga}}$ split. ${ }^{11-13}$ This model provides a good description of the electrical recovery mechanism but does not give information about the photoinduced recovery from metastable EL2. To explain this process observed at energies between 1.4 and $1.5 \mathrm{eV}$, Manasreh and Fisher ${ }^{8}$ proposed the $A s_{\mathrm{Ga}}-\mathrm{V}_{\mathrm{As}}$ model. Finally, an extensive discussion about the presence of arsenic and gallium vacant defects in the microscopical structure of EL2 family and their influences in the optical and electrical characteristics has been reported by Wager and Van Vetchen. ${ }^{42,45}$

From our results, and according to the above considerations, we propose that the existence of an EL2 photoinduced recovery process in semi-insulating, oxygen-doped and boron- and oxygen-implanted samples could be related basically to the interaction between the basic EL2 ( $\mathrm{As}_{\mathrm{Ga}}$ antisite) and an arsenic vacancy defect and/or gallium vacancy. The implantation process can assist the complex defect creation from this simple defect, and that would explain the changes in the metastable optical recovery observed. The similar vacancy distribution for the oxygenand boron-implanted samples could explain the similar optical recovery spectral dependence. Assuming this hypothesis, for the oxygen-doped samples, this atom would increase the vacancy defect concentration and help its interaction with the $A s_{G a}$ defect. Nevertheless, more experiments will be necessary to determine an accurate microscopic model.

\section{CONCLUSIONS}

In summary, we have analyzed the voltage phototransients obtained at constant capacitance conditions from boron- and oxygen-implanted samples using optical isothermal transient spectroscopy. The results point out that the ion implantation process creates EL2-like defects, which interact with shallower levels also produced by the implantation. The created EL2 presents a partial or complete inhibition of the electrical recovery. This behavior does not depend on the implanted specimen (boron or oxygen) and is clearly related to the presence of damage defects. We have also determined the existence of an optical induced recovery process from the metastable state competing with the photoquenching effect in the spectral range 1.1-1.4 eV. The spectral dependence of this optical recovcry has been found to be basically independent of the ion specimen, dose, and annealing for temperatures below $450^{\circ} \mathrm{C}$. Finally, a model is suggested for the EL2 defect which presents optical recovery consisting of a complex that involves $\mathrm{As}_{\mathrm{Ga}}$ and gallium and/or arsenic vacancies.

${ }^{1}$ G. M. Martin and S. Makram-Ebeid, Deep Centers in Semiconductors, edited by S. T. Pantelides (Gordon and Breach, New York, 1986), p. 399.

${ }^{2}$ J. C. Bourgoin, H. J. Bardeleben, and D. Stievenard, J. Appl. Phys. 64, R65 (1988).

${ }^{3}$ G. Guillot, Rev. Phys. Appl. 23, 833 (1988).

${ }^{4}$ Y. Mochizuki and T. Ikoma, Jpn. J. Appl. Phys. 24, L895 (1985).

${ }^{5}$ S. Nojima, J. Appl. Phys. 58, 3485 (1985).

${ }^{6}$ D. W. Fisher, Appl. Phys. Lett. 50, 175 (1987).

${ }^{7}$ M. Tajima, H. Sauto, T. Tino, and K. Ishid, Jpn. J. Appl. Phys. 27, 4101 (1988). 
${ }^{8}$ M. O. Manasreh and D. W. Fisher, Phys. Rev. B 40, 11756 (1989).

${ }^{9}$ H. J. von Bardeleben, D. Stievenard, D. Deresmes, A. Huber, and J. C. Bourgoin, Phys. Rev. B 34, 7192 (1986).

${ }^{10}$ B. K. Meyer, D. M. Hofman, J. R. Niklas, and J. M. Spaeth. Phys. Rev. B 36, 1332 (1987).

${ }^{11}$ M. Kaminska, M. Skowronski, and W. Kuszks, Phys. Rev. Lett. 55, 204 (1985).

${ }^{12}$ D. J. Chadi and K. J. Chang, Phys. Rev. Lett. 60, 2187 (1988).

${ }^{13} \mathrm{~J}$. Dabrowski and M Scheffer, Phys. Rev. Lett. 60, 2183 (1988).

${ }^{14}$ J. C. Parker and R. Bray, Phys. Rev. B 37, 6368 (1988).

${ }^{15}$ U. V. Desnika, D. I. Desnika, and B. Santic, Appl. Phys. Lett. 58, 278 (1991).

${ }^{16}$ T. R. Jervis, D. W. Woodard, and L. F. Eastman, Electron. Lett. 16, 621 (1976).

${ }^{17}$ M. Taniguchi and T. Ikoma, Inst. Phys. Conf. Ser. 65, 65 (1983).

${ }^{18}$ G. M. Martin, P. Terriac, S. Makram-Ebeid, G. Guillot, and M. Gavand, Appl. Phys. Lett. 42, 61 (1983).

19 J. R. Morante, A. Pérez, J. Samitier, and A. Romano. J. Appl. Phys. 70, 4202 (1991).

${ }^{20} \mathrm{~J}$. Samitier, J. R. Morante, A. Cornet, A. Herms, P. Roura, and A. Pérez, Mater. Sci. For. 10-12, 539 (1986).

${ }^{21}$ A. Chantre, G. Vincent, and D. Bois, Phys. Rev. B 23, 5335 (1981).

${ }^{22}$ G. Vincent, D. Bois, and A. Chantre, J. Appl. Phys. 53, 3643 (1982).

${ }^{23}$ S. W. Provenchar, Compt. Phys. Commun. 27, 213 (1982).

${ }^{24} \mathrm{~K}$. Ikeda and H. Takaoka, J. Appl. Phys. 54, 6031 (1983).

${ }^{25}$ I. Isenberg, R. D. Dyson, and R. Hanson, Biophys. J. 13, 1090 (1973).

${ }^{26} \mathrm{~S}$. Marco, J. Samitier, J. M. López-Villegas, and J. R. Morante, Meas. Sci. and Tech. 2, 899 (1991).

${ }^{27}$ J. Samitier, J. R. Morante, L. Giraudel, and S. Gourrier. Appl. Phys. Lett. 48, 1138 (1986).

${ }^{28}$ A. Pérez, J. Samitier, J. Esteve-Tinto, A. Romano, and J. R. Morante, Defect and Diffusion Forum 62/63, 77 (1989).

${ }^{29}$ S. Marco, J. Samitier, J. R. Morante, P. Boher, and M. Renaud, in Proceedings of the 6th International Conference on Semi-Insulating III-
$V$ Materials, Toronto 1990 , edited by A. G. Milnes and C. Miner (Adam Hilger, Bristol, 1990).

${ }^{30}$ D. M. Hofman, B. K. Meyer, J. M. Spaeth, M. Wattenbach, J. Krüger, C. Kisielowski-Kemmerich, and H. Alexander, J. Appl. Phys. 68, 3381 (1990).

${ }^{31}$ G. M. Martin, E. Esteve, P. Langlade, and S. Makram-Ebeid, J. Appl. Phys. 56, 2655 (1984).

${ }^{32}$ J. R. Morante, J. Samitier, A. Pérez, H. Altelarrea, and S. Gourrier, J. Appl. Phys. 60, 1661 (1986).

${ }^{33}$ X. Boddaert, D. Stievenard, and J. C. Bourgoin, Phys. Rev. B 40, 1051 (1989).

${ }^{34}$ M. Taniguchi, T. Ikoma, Proc. 2nd Conf. Semi-Insulating III-V Materials, Evian, 1982, edited by S. Makram-Ebeid and B. Tuck, p. 283.

${ }^{35}$ J. Samitier, J. R. Morante, A. Miralles, A. Cornet, A. Herms, and S. Gourrier, Proc. 4th Conf. on Semi-Insulating III-V Materials, Hakone 1986, p. 579.

${ }^{36}$ S. Makram-Fheid and P. Boher, Rev. Phys. Appl. 23, 847 (1988).

${ }^{37}$ X. Zhong, D. Jiang, W. Ge, and C. Song, Appl. Phys. Lett. 52, 628 (1988).

${ }^{38}$ J. Schneider, B. Dischler, H. Seelewind, P. M. Mooney, J. Lagowski, M. Matsuni, D. R. Beard, and R. C. Newman, Appl. Phys. Lett. 54, 1442 (1989).

${ }^{39}$ J. C. Bourgoin, D. Stievenard, D. Deresmes, and J. Mimila-Arroyo, J. Appl. Phys. 69, 284 (1991).

${ }^{40}$ J. Lagowski, D. G. Lin, T. Aoyama, and H. C. Gatos, Appl. Phys. Lett. 44, 336 (1984).

${ }^{41}$ S. T. Neild, M. Skowronski, and J. Lagowski. Appl. Phys. Lett. 58, 859 (1991).

${ }^{42}$ J. F. Wager and J. A. Van Vetchen, J. Appl. Phys. 62, 4192 (1987).

${ }^{43}$ P. Dansas, J. Appl. Phys. 58, 2212 (1985).

${ }^{44}$ G. M. Martin, P. Secordel, and C. Verger, J. Appl. Phys. 53, 8706 (1982).

${ }^{45}$ J. F. Wager and J. A. Van Vetchen, Phys. Rev. B 35, 2330 (1987). 